\title{
A Chance Favors a Prepared Mind: Chance Discovery from Cognitive Psychology
}

\author{
Hitoshi Terai and Kazuhisa Miwa \\ Graduate School of Information Science, Nagoya University, \\ Furou-chou, Chikusa-ku, Nagoya, Aichi, Japan \\ \{terai,miwa\}@is.nagoya-u.ac.jp \\ http://miwalab.cog.human.nagoya-u.ac.jp
}

\begin{abstract}
A chance has two contrary aspects: suddenness as an accidental finding and gradualness as the result of a prepared mind. Such duality of chance discovery resembles the insight process treated by problem solving researches. In this paper, we focus on the insight process in human problem solving, present a broad overview of its suddenness and the gradualness, and introduce our experimental results from the viewpoint of the duality of insight. We believe that our research findings will contribute to studies of chance discovery.
\end{abstract}

Keywords: aha experience, constraint relaxation, eye movement, impasse, incubation, insight, prepared mind, reconstruction, representation change

\section{Introduction}

In the study of chance discovery, a chance is defined as an event or situation that may crucially affect decision-making or suggest that an important event will occur in the future. The studies of chance discovery address how we can exploit such chance to benefit the development of a new market, to avoid risk, etc.

Such aspects are not new ways of thinking about chance discovery. In data mining, methodologies that reveal the tendencies of past data and predict the future have been investigated for many years. The originality of the study of chance discovery is in the perspective of a chance. The word "chance" comes from the Latin casus, meaning luck. It means that one has the impression that a chance discovery arises from uncontrollable causes. Creators who noted important historical things often reported that their findings were blessings [4]. Such perspectives about chance discovery stress that there is no preparation to find a chance.

On the other hand, consider the proverb: "Chance favors a prepared mind." This perspective recognizes that chance is related to human factors. Where do these contradictions come from? Perhaps a prepared mind was unconsciously processed. Such duality of chance discovery, suddenness as an accidental finding 
and gradualness as a process of a prepared mind, resembles the insight process treated by problem solving researches.

In the paper, we focus on the insight process in the study of human problem solving, give a broad overview of the suddenness and the gradualness of insight, and introduce our experimental results from the viewpoint of the duality of insight.

\section{Insight Problem Solving}

In the Gestalt psychology conception, thinking is mainly classified as either reproductive or productive. Reproductive thinking is the application of previously acquired knowledge to a problem. On the other hand, productive thinking is the ability to go beyond experience and produce something new in response to the demands of a problem. Insight problem solving is categorized as the latter[10, 26].

Insight problem solving is different from normal problem solving in many aspects[21]. In Gestalt psychology, the insight process is defined as follows. First, configuration of a problem and past experience prevent it from finding the relations required to solve a problem, creating an impasse for the problem solver. Finally, insight may arise through the reconstruction of a problem from a different perspective[16,10].

Box 1 shows an example of an insight problem that requires reconstruction of an incorrect recognition of the problem. As long as the problem solver treats it superficially, he or she cannot solve it. The problem's structure must be reconstructed to find the covert relations. In the problem, only the circle's radius is given; the diagonal's length cannot be obtained directly. However, the solution can be obtained through the following reconstruction. A rectangle has two diagonals. By focusing on the second diagonal, the problem solver realizes that it equals the rectangle (aha!). Thus, when the perspective of a problem is reconstructed, a solution is derived from that reconstruction result $[8,11]$.

Box 1 Geometrical Problem

If the length of the radius is $r$, what is the length of line $l$ ?

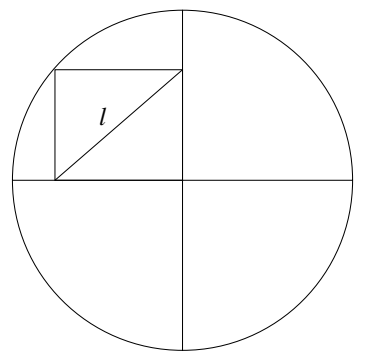


Six-year-old Karl Gauss solved a tedious arithmetic problem very quickly by reconstructing it[14]. Hoping to give himself a few minutes' peace, his teacher asked his class to add up the numbers $1+2+3+4$ etc. up to 100. Hardly had the class begun to add up all the numbers when Gauss put his hand up with the answer: 5050. Gauss had reconstructed the problem. He made two numerical sequences: an ascending sequence from 1 to 100 and a descending sequence from 100 to 1 . He could make a hundred 101 by adding the numbers at the same position on each sequence $(1+100,2+99$, etc. $)$. Adding the series $1+2+$ 3 up to 100 is therefore the same as taking half of 10, 100; that is 5050. Thus, reconstruction occurs not only in perceptual relations but also in conceptual relations.

\section{Duality of Insight Process}

The moment of insight problem solving by reconstructing the problem seems to occur suddenly. On the other hand, a preparation phase has been identified during insight problem solving. In this section, we focus on such contradictory aspects, suddenness and gradualness, in the process of insight problem solving.

\subsection{Suddenness of Insight}

In the process of insight problem solving, a solution that seems to arise suddenly is generally known as an "aha! experience." Such suddenness, a key feature of insight problem solving, is also found in the recollections of historical scientific findings [25].

Metacognition in Insight Problem Solving Metcalfe and Wiebe discussed the suddenness of insight problem solving from the viewpoint of metacognition by focusing on it both before and during insight problem solving[12]. In their experiment, participants were given both insight and non-insight problems and were required to rearrange them into a line going from the problem they thought they were most likely to solve to the one they were least likely to solve. The correlation results between the difficulty of the problems and the response time of each revealed that the participants fairly accurately predicted which noninsight problems they would be able to solve later, but they were unable to predict which insight problems they would solve.

Moreover, every 15 seconds while solving the problems, the participants were told to indicate their feeling of warmth (i.e., their perceived closeness to a solution). The result shows that the patterns of warmth ratings, which refract participant feelings about the approaching solution, differed for insight and noninsight problems. Non-insight problems showed a more incremental pattern than the insight problems. In general, the data indicate that non-insight problems were open to accurate predictions of performance, but the insight problems were opaque to such predictions. 
Metcalfe and Wiebe's findings were as follows. The first is about metacognition prior to addressing insight problems. People can accurately predict the degree of difficulty of non-insight problems before solving them, but not in insight problem solving. The second is about metacognition in insight problem solving. People can accurately recognize a differential between the current state and the goal state in non-insight problems, but they cannot do so in insight problem solving; they feel that they are suddenly finding a solution.

\section{Box 2 Box-Candle Problem}

You have a candle, matches, and a box of tacks. Support the candle on the wall without dripping wax on the table.

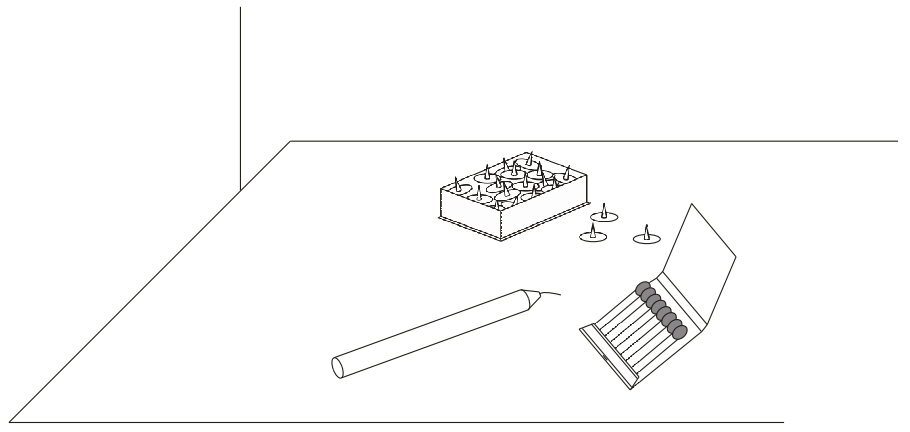

Representation Change Furthermore, aspects of problems such as manner of formulation of problems change fundamentally across before and after finding a solution [11]. For example, in the box-candle problem (Box 2), a famous task used in studies of insight problem solving, subjects are given candles, matchsticks, and a box filled with tacks. The objective of the task is to attach a candle to the wall so that it will not drip wax on the table. Using the box filled with the tacks is essential to solve this problem. However, despite the fact that the solution of this problem is very simple, finding the solution is difficult because we embrace the illusion that the box filled with tacks must hold the tacks as a container (see Appendix for the solution). Such an assumption is automatically established; reconstructing the incorrect structure of problems is required to find solutions $[10,16]$. After establishing such reconstruction, if in the future the same problem is encountered, the impasse will not be met again.

These studies revealed that in insight problem solving, we cannot estimate current problem solving states and feel that representation suddenly changes 
with flash of illuminance at the moment when finding a solution. Such suddenness in the problem solving process is a crucial characteristic of insight problem solving.

\subsection{Gradualness of Insight}

In contrast to the sudden attainment of a solution described above, the process of insight problem solving develops through a prepared phase. For example, you experience an impasse, and you forget about the problem for a while, and then a solution suddenly appears while you are thinking about other things. Actually, many prominent creative individuals have reported similar introspective reports[25].

Incubation Wallas outlined four major phases of information processing that may mediate innovative problem solving and creativity: (1) mental preparation, (2) incubation, (3) illumination, and (4) verification[25,17]. The initial preparation phase confronts an important problematic situation, conceptualizes the problem's core aspects, and makes exerted tentative unsuccessful attempts to reach a satisfactory resolution. Next, the incubation phase puts the problem aside and instead thinks about other matters for an extended period of time. Then, at some point during the incubation, there is a sudden shift to the illumination phase, where a penetrating flash of insight unexpectedly occurs about an appropriate satisfying resolution to the original problematic situation. Given the attained insight, the final verification phase concentrates on the details of the resolution or determines that it was applied successfully.

Next, we introduce two major studies about the effect of the incubation phase during solving insight problems. Silveira showed its effect by conducting psychological experiments $[18,17]$ in which the participants were given the necklace problem shown in Box 3 (see appendix for the solution). They were instructed to put the problem aside as an incubation phase during problem solving; they resumed solving the problem after the incubation phase. The interruption time was controlled as an experimental factor.

The experiment results showed that $64 \%$ and $85 \%$ of the participants in the experimental conditions with short $(30 \mathrm{~min})$ and long $(4 \mathrm{~h})$ incubation phases found the solution, whereas only $55 \%$ of the participants in the control condition found the solution without the incubation phase.

Smith and Blankenship examined the effect of fixation and incubation in the insight process using remote associate tests (RAT)[20]. Box 4 shows an example of a RAT problem (see appendix). In the experiments, there were two experimental factors: fixation (fixation/nonfixation), and incubation (incubation/noincubation). For RAT problems, the participants must think of a word that in combination with each of the three test words makes a two-word phrase or a compound word. For example, the solution to the RAT problem "TYPE / GHOST / STORY" is "WRITER." To lead the participants to the initial fixation, words were given as misleading clues (in this example, "STYLE / GOBLIN / TALE" were given at the same time). 
Box 3 Necklace Problem

You have four separate pieces of chain that are each three links long, as shown on the left side of the figure. It costs 2 cents to open a link and 3 cents to close a link. All links are closed at the beginning of the problem. Your goal is to connect all 24 links into a single circle, as shown on the right side of the figure. The total cost must not exceed 15 cents.

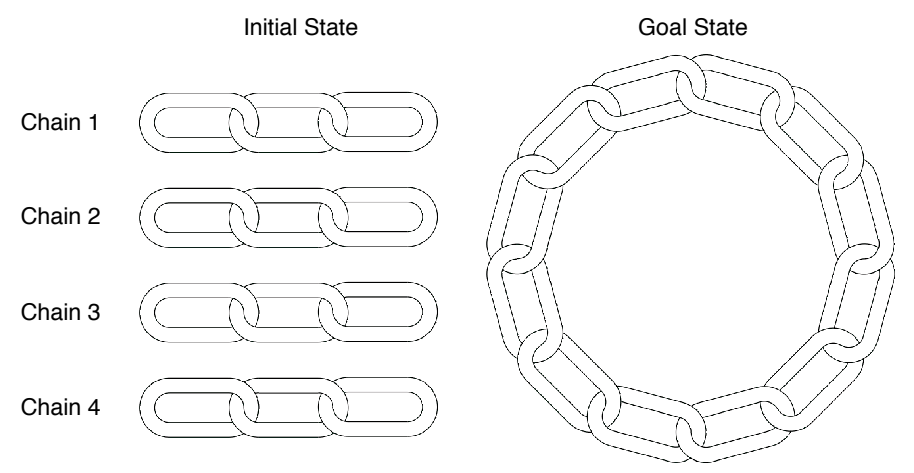

The results of the experiments revealed that incubation significantly facilitated problem solving in the fixated group, but there was no significant effect in the non-fixated group.

Incubation unrelated to the confronted problems affected improvements to the problem solving and indicate that there is a preparation process that is different from conscious effort; such a process has a significant role in problem solving. In the next section, we discuss this unconscious process of a prepared mind from the viewpoint of the relaxation of mental constraints.

\section{Box 4 Remote Associate Test}

Find a single word that forms a common word or phrase with each of the three words in the upper row.

$$
\begin{array}{llc}
\text { TYPE } & \text { GHOST } & \text { STORY } \\
\text { style } & \text { goblin } & \text { tale }
\end{array}
$$


Mental Constraint Relaxation In some cases the structure of problems and previous knowledge act as negative constraints and inhibit problem solving. Therefore problem solvers may encounter an impasse around which they can't proceed. Box 5 shows the nine dots problem, which is one of the most famous experimental tasks in the study of insight problem solving (appendix).

Even though this problem is seemingly very simple, many people cannot solve it. The difficulty arises from an unconscious, incorrect assumption that the lines should begin and end on dots and were drawn within a square made by eight dots outside of the nine $\operatorname{dots}[9,16,15,2]$.

Box 5 Nine Dots Problem

Connect all nine dots using four straight lines without lifting your pen.

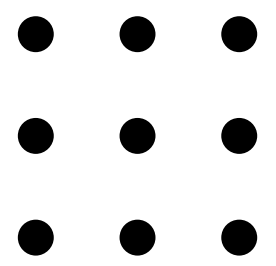

Recently, the insight process has been described from the viewpoint of mental constraint relaxation. First, mental constraints arise from past experiences and/or the structures of problems, and the problem solver meets an impasse because these constraints prevent a solution from being reached. Such mental constraints mean the false formulation of problem structure and incorrect interpretation of problems. Through problem solving, these mental constraints are gradually relaxed. A search that does not follow these mental constraints gradually increases, and then the problem solver reaches a solution [6, 13].

Knoblich et al. analyzed mental constraints using the matchstick arithmetic problem that consists of a false arithmetic statement written with roman numerals (I, II, III, etc.), arithmetic operation (+, -), and equal signs constructed out of matchsticks[7]. Box 6 shows an example that was presented to the participants in their study. The goal is to move a single matchstick so that the initial false statement is transformed into a true arithmetic statement (appendix).

In their study, they argued that impasses are broken by changing the problem representation and described two hypothetical mechanisms for representational change: the relaxation of the constraints of the solution and the decomposition of perceptual chunks.

Problem solvers must relax one or more of three constraints: value, operator, and tautology. When mapped onto three representational levels, the three constraints each have different scopes. When the value constraint is relaxed, 
the problem solver can change a numerical value to another (e.g., from "IV" to "VI"). When the operator constraint is relaxed, the problem solve can change an arithmetic function to another (e.g., from "+" to "="). When the tautology constraint is relaxed, the problem solver can change an arithmetic statement to a tautological statement (e.g., from "III = III + III" to "III = III = III").

On the other hand, familiarity with a class of objects or events creates patterns that capture the recurring constellations of features or components. Knoblich et al. proposed that the probability that a chunk will be decomposed is inversely proportional to its tightness. For example, the problem solver can decompose "VI" to "V" and "I" more easily than "V" to a pair of "I"s because the former's chunk is loose, but the latter's is tight. They concluded that a constraint with a wide scope is more difficult to relax than one with a narrow scope. A tight chunk is more difficult to decompose than a loose one, and constraints and chunks independently lead the problem solver to an impasse.

Box 6 Matchstick Arithmetic Problem

Transform the false equation into a true equation by moving a match.

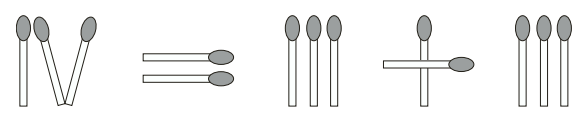

\section{Experiment}

As described above, previous researches either highlighted the suddenness or the gradualness of the nature of the insight process. However, two contrary natures of the insight process should be understood as its bilateral character. Until insight is consciously perceived as a sudden event, the preparing process should proceed unconsciously. However little research has addressed such duality of insight.

In this section, we introduce our study that revealed the duality of the insight problem solving.

\subsection{Dual Space Search}

We investigated the process of insight problem solving using a discovery task. The discovery process is the interaction between searching behavior for hypotheses held by the problem solver and instances in the environment used to construct 
and test them. Simon and Lea discussed such a discovery process as the interaction between searching for a rule space and an instance space[19]. Such an aspect has been established as a dual space search model of the scientific discovery process by conducting many psychological experiments and constructing computational models[5].

In our study, we conducted a psychological experiment to capture the duality of the insight process based on the dual space search model. To analyze the insight process as a dual space search, searching for a mental problem space is defined as searching for hypothesis space, and gathering data during hypothesis formation and testing is defined as searching for data space.

Verbal Protocole In our task, since the participants reported their hypotheses, we captured the process of their hypothesis searches as verbal reports.

Eye movement We also captured their searching for data as eye movements.

\subsection{Method}

Subjects Twenty-four undergraduate students participated in this experiment.

Task In this study, we proposed and used a discovery task that asked subjects to find a rule for predicting a digit. In the task, subjects must find a target rule as a solution through hypothesis formation and testing.

An example screenshot of the task is shown in Fig. 1. The display consists of three slots, and in each slot a single digit rotates at a speed that prevents the subjects from perceiving each digit. A history data window indicates the past four trials. The digit in the third slot is controlled by an unknown rule (target rule). If subjects find the target rule, then they can predict the digit in the third slot. The mission of the subjects is to find the target rule and predict the digits in the third slot.

Subjects are required to predict the digit in the third slot after the two digits in the first and second slots stop rotating. A series of procedures, which includes stopping the first and second slots and then predicting and confirming the third digit, is called a trial; trials are repeated until subjects find the target rule. A history data window below the three slots shows the results of the past four trials.

The rules reported in each trial by subjects are called hypotheses, which are proposed in the process of hypothesis formation and testing. This is a discovery task that requires insight. Therefore, the task is manipulated to lead the subjects to find a sham rule called a blocking hypothesis, which differs from the target rule. The target rule and blocking hypothesis are shown below. The target rule states: "the third digit in the nth trial is determined by adding three to the third digit in the n-1th (previous) trial" with a vertical relation on the display. The blocking hypothesis states: "the third digit is equal to the sum of the first and second digits" with a horizontal relation. 


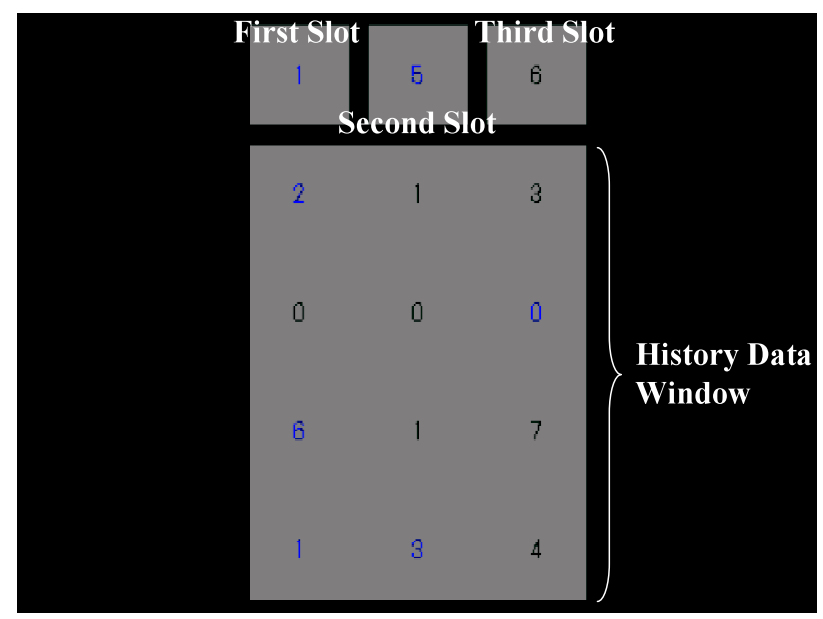

Fig. 1. Example screenshot of discovery task used in this study.

Subjects are required to predict the digit in the third slot after the two digits in the first and second slots stop rotating. In the initial eight trials, by controlling the first and second digits, the third digit is consistent with the sum of the first and second digits while maintaining the target rule across the vertical row (see example display in Fig. 1 ; this rule is confirmed as follows: $1+3=4,6+1=$ $7,0+0=0,2+1=3,1+5=6$ in each column as a blocking hypothesis, and a series of 4, 7, 0, 3, 6 from bottom to top in the third row as the target rule). Therefore, subjects are guided to form a blocking hypothesis with the horizontal relation as a sham rule. After the first eight trials, a digit predicted by the blocking hypothesis gradually disagrees with an actual third digit. That is, the blocking hypothesis doesn't predict the third digits but the target rule is still maintained. Consequently, from the ninth trial subjects begin to receive negative instances called negative feedback that disconfirms the blocking hypothesis.

Definition of Types of Hypothesis Spaces The process through which subjects discover the target rule is considered a process of searching for a hypothesis space. A group of hypotheses that share a common regularity constitutes a hypothesis space; therefore the target rule and the blocking hypothesis belong to different problem spaces. In this study, the relation between hypotheses and hypothesis spaces is defined as follows:

Blocking Hypothesis Space: A set of hypotheses characterized by a horizontal relation is defined as a blocking hypothesis space.

Blocking Hypothesis: described above.

Horizontal Hypotheses: rules characterized by a horizontal relation other than the blocking hypothesis (e.g., " the third digit is equal to the subtraction of the 


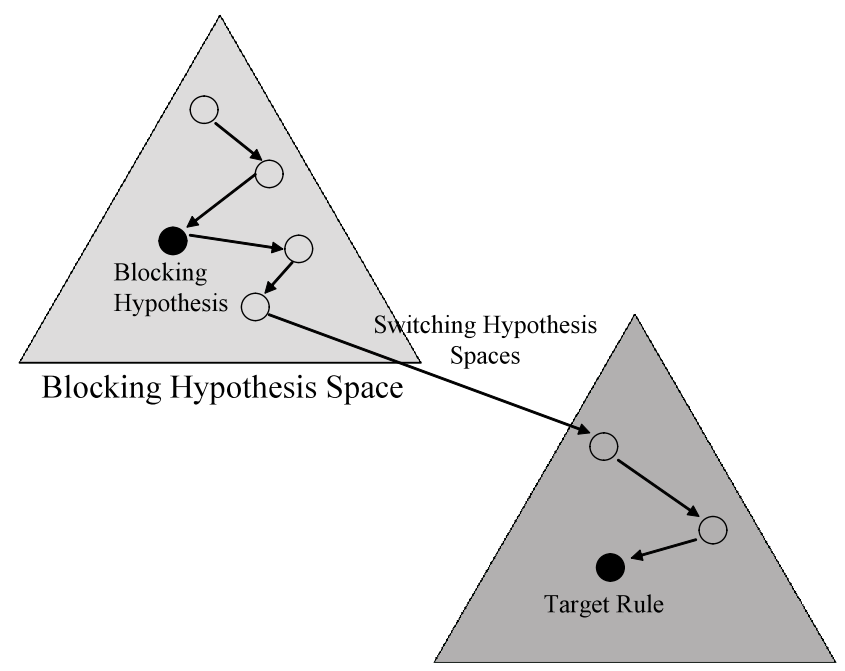

Target Space

Fig. 2. Relation between Blocking Hypothesis and Target Rule.

second digit from the first digit"). Target Space A set of hypotheses characterized by a vertical relation is defined as a target space.

Target Rule: described above.

Vertical Hypotheses: rules characterized by a vertical relation other than the target rule (e.g., "the third digit is equal to the sum of the third digits in all past trials in the history data window"). In addition to these hypotheses, subjects reported hypotheses characterized by both horizontal and vertical relations, which are involved neither in the blocking hypothesis space nor in the target space, such as identical digits arranged diagonally.

The search for each hypothesis space described above is demonstrated in Fig. 2. Running into an impasse and finding a target rule are shown as follows when the insight process is considered a search for problem spaces. An impasse means that after finding a blocking hypothesis, subjects are fixated on searching for the blocking hypothesis space that consists of similar hypotheses with the horizontal relation to the blocking hypothesis, even if the blocking hypothesis is disconfirmed. It is necessary to exit from the search for the blocking hypothesis space and move to a search for the target space to find the target rule. A previous study indicated that once the blocking hypothesis was formed, many subjects could not extricate themselves from incorrect constraints and failed to find the target rule even though it is very simple.

Procedure Subjects start a trial, report a predicted rule as a hypothesis after the first and second slots stopped, and discontinue the third slot to confirm the 
hypothesis. Such trials consisting of hypothesis proposing and testing lasted for a maximum of 55 minutes until finding the target rule. If subjects reported the target rule continuously five times, then it was considered that they had found the target rule.

\subsection{Results}

We excluded eight of the twenty-four subjects from analysis because they could not form a blocking hypothesis during the initial eight trials or could not provide any fine eye movement data. Five of the sixteen subjects found the target rule and were categorized as the successful group; eleven subjects did not find the target rule and were categorized as the unsuccessful group. In this paper, we are only concerned with the results of the successful group (for details, see [23]).

Transition of Hypothesis Space Search The proposed hypotheses were categorized into one of the five types defined in section 4.2 ; how they changed was also analyzed. In this experiment, we did not provide a second evaluater because in each trial the subjects were required to verbally report proposed hypotheses, which we clearly captured. Fig. 3 shows the transition of the proposed hypotheses in the successful group. The horizontal axis indicates the number of trials, and the vertical axis indicates each type of hypothesis and the hypothesis space described above. On the vertical axis in Fig. 3, the blocking hypothesis and hypotheses with horizontal relations correspond to hypotheses within the blocking hypothesis space in Fig. 2, and the target rule and hypotheses with vertical relations correspond to hypotheses within the target space in Fig. 2.

Fig. 3 shows that all successful subjects found the blocking hypothesis by the time they reached the ninth trial. After the ninth trial, they began to receive negative instances for the blocking hypothesis and to propose other hypotheses than the blocking hypothesis. However, hypotheses outside of the blocking hypothesis space were almost never proposed, confirming that the subjects continued to search for the blocking hypothesis space. This result indicates that the subjects had run into an impasse. Fig. 3 also shows that discovering the target rule suddenly seemed to occur while escaping from a state where subjects searched for the blocking hypothesis space, rather than through gradual shifting of potential hypothesis spaces. For example, subject I5 suddenly found the target rule from the fourteenth to fifteenth trials with an abrupt shift from searching for the blocking hypothesis space to the target space. Correspondingly, the other successful subjects also found the target rule suddenly after abandoning their search for the blocking hypothesis space.

Transition of Data Space Search Next, we analyze the process of subjects searching for data spaces that led to finding the target rule using eye movement data, obtained as transition patterns of the fixation of eye movement. In this study, eye movements from nth to $\mathrm{n}+1$ th fixation points were categorized as $(1)$ horizontal, (2) vertical, (3) diagonal, or (4) fixed. 
The transition of the horizontal and vertical eye movements of successful subjects is shown in Fig. 4. The horizontal axis shows the number of trials: the first nine trials, three trials after negative feedback was given, and four trials before and after the target rule was discovered. The vertical axis indicates the ratio of each type of eye movement to all types of eye movement (horizontal, vertical, diagonal, and fixed). Fig. 4 shows that the horizontal eye movement, corresponding to searching for the data space correspond to the blocking hypothesis space, dominated until the subjects reached the ninth trial. By contrast, after

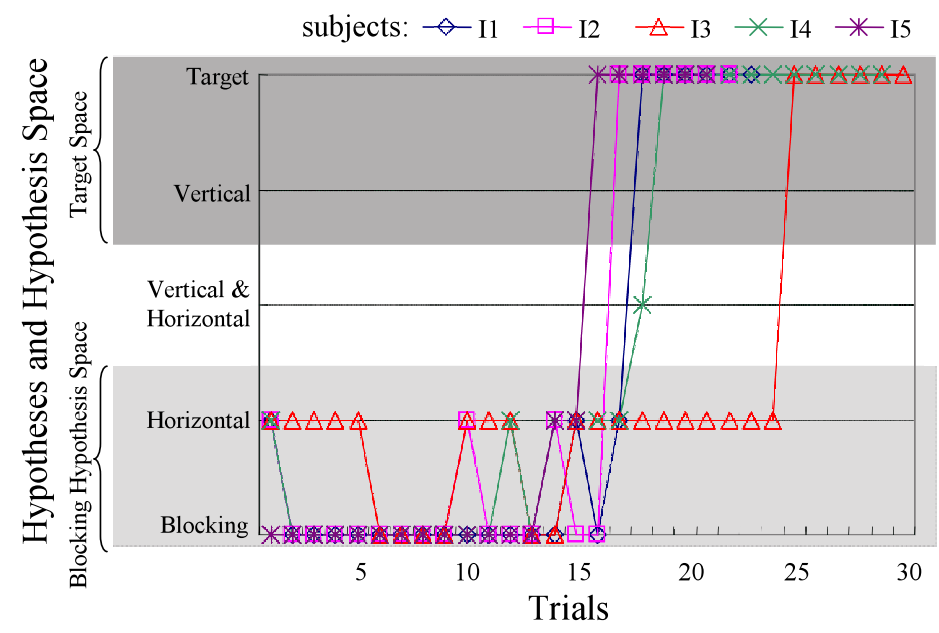

Fig. 3. Transition of hypothesis search in successful groups.

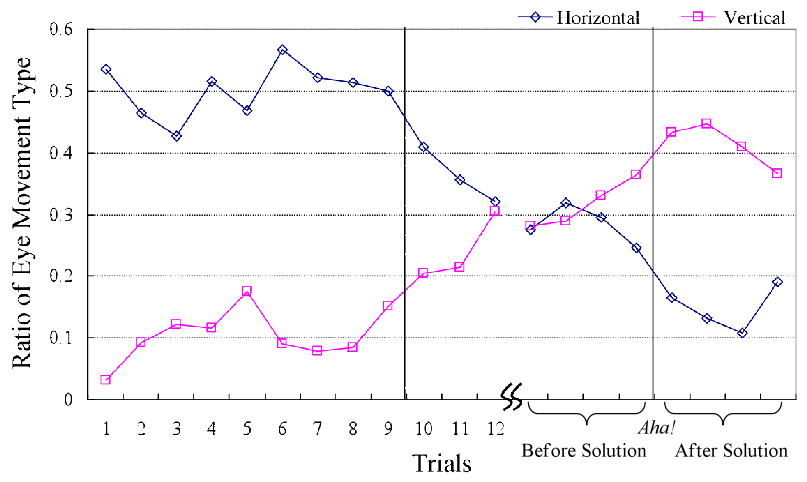

Fig. 4. Transition of data search in successful groups. 
they were given negative feedback, the ratio of horizontal eye movement gradually decreased but the ratio of vertical eye movement gradually increased. This result indicates that the search for the data space correspond to the blocking hypothesis space gradually decreased and the search for the data space correspond to the target space gradually increased. The gradual shift of searching for data spaces in Fig. 2 was captured in subject eye movement data.

\subsection{Summary}

In psychological experiments, we captured the transitions of hypothesis space search and data space search in the process of hypothesis formation and testing using subjects' verbal reports and eye movement analysis. From the viewpoint of the former, regarding their hypotheses as hypothesis space search, discovering the target rule seemed to occur suddenly following an impasse. From the viewpoint of the transition of the proposed hypotheses the experimental result showed exactly that in insight problem solving, a solution is found with flash of a illuminance called the "aha experience" that characterizes the suddenness of the insight process $[1,12]$. On the other hand, the analysis of subject eye movement as data space search revealed that searching for hypotheses gradually varied with the development of problem solving after negative feedback was given.

\section{Conclusion}

In this paper, we argued that in insight problem solving tasks, a prepared mind precedes the "aha experience". Such a process of reaching insight resembles the requirements of chance discovery. How do you help and lead a prepared mind to chance discoveries? Many challenges remain. To answer these questions, we must develop interdisciplinary studies between cognitive researches about understanding what is chance for humans and information engineering about specific techniques that encourage chance discovering.

Moreover, we introduced our research that attempted to reveal the prepared process of insight problem solving using eye movement data. A more direct way to capture unconscious processes is measuring brain activity. In recent years, such non-invasive brain imaging apparatus as Near InfraRed Spectroscopy (NIRS) and functional Magnetic Resonance Imaging (fMRI) have been developed. Future developments are expected to study the effective use of these measurement techniques. In addition, another approach is to construct a computational model that may represent the interaction of conscious and unconscious processes to explain the duality of the insight process. In problem solving research, there are already related studies (e.g., [22]). We have already begun a study based on a computational model approach[24]. 


\section{References}

1. J. E. Davidson. The suddenness of insight. In R. J. Sternberg and J. E. Davidson, editors, The nature of insight, chapter 4, pages 125-155. Cambridge, MA: MIT Press., 1995.

2. R. L. Dominowski and P. Dallob. Insight and problem solving. In R. J. Sternberg and J. E. Davidson, editors, The nature of insight, chapter 2, pages 33-62. Cambridge, MA: MIT Press., 1995.

3. K. Duncker. On problem-solving. Psychological Monographs, 58(270):1-113, 1945.

4. P. Ferrucci. Inevitable Grace: Breakthroughs in the Lives of Great Men and Women : Guides to Your Self-Realization. St Martins Pr, 1990.

5. D. Klahr and K. Dunbar. Dual space search during scientific reasoning. Cognitive Psychology, 12:1-55, 1988.

6. G. Knoblich, S. Ohlsson, and Raney G. E. An eye movement study of insight problem solving. Memory \&3 Cognition, 29(7):1000-1009, 2001.

7. G. Knoblich, S. Ohlsson, H. Haider, and D. Rhenius. Constraint relaxation and chunk decomposition in insight problem solving. Journal of Experimental Psychology: Learning, Memory, and Cognition, 25(6):1534-1555, 1999.

8. W. Kohler. The task of Gestalt psychology. Princeton, NJ: Princeton University Press, 1969.

9. N. R. F. Maier. Reasoning in humans: I. on direction. Journal of Comparative Psychology, 10:115-143, 1930.

10. R. E. Mayer. Gestalt -thinking as restructuring problems-. In Thinking, problem solving, cognition, chapter 3, pages 39-78. New York, W.H. Freeman and Co., 2nd edition, 1992.

11. R. E. Mayer. The search for insight: Grappling with gestalt psychology's unanswerd questions. In R. J. Sternberg and J. E. Davidson, editors, The nature of insight, chapter 1, pages 3-32. Cambridge, MA: MIT Press, 1995.

12. J. Metcalfe and D. Wiebe. Intuition in insight and noninsight problem solving. Memory \& Cognition, 15(3):238-246, 1987.

13. S. Ohlsson. Information-processing explanations of insight and related phenomena. In M. Keane and K. Gilhooley, editors, Advances in the psychology of thinking chapter 1, pages 1-44. Harvester Wheatsheaf: London, 1992.

14. S. I. Robertson. Problem solving. Taylor \& Francis, 2001.

15. D. Rumelhart. Introduction to human information processing. New York: Wiley, 1977.

16. M. Scheerer. Problem solving. Scientific American, 208:118-128, 1963.

17. C. M. Seifert, D. E. Meyer, N. Davidson, A. L. Patalano, and I. Yaniv. Demystification of cognitive insight: Opportunistic assimilation and the prepared-mind perspective. In R. J. Sternberg and J. E. Davidson, editors, The nature of insight, chapter 3, pages 65-124. Cambridge, MA: MIT Press, 1995.

18. J. M. Silveira. Incubation: The effect of interruption timing and length on problem solution and quality of problem processing. Unpublished doctoral dissertation, University of Oregon, Eugene, 1971.

19. H. A. Simon and G. Lea. Problem solving and rule induction: A unified view. In L. Gregg, editor, Knowledge and Cognition. Hillsdale, N. J.: Erlbaum, 1974.

20. S. M. Smith and S. E. Blankenship. Incubation and the persistence of fixation in problem solving. American Journal of Psychology, 104:61-87, 1991.

21. R. J. Sternberg and J. E. Davidson, editors. THE NATURE OF INSIGHT. Cambridge, MA: MIT Press, 1995. 
22. R. Sun. DUALITY of the MIND. LAWRENCE ERLBAUM ASSOCIATES, PUBLISHERS, Mahwah, New Jersey, London, 2002.

23. H. Terai and K. Miwa. Insight problem solving from the viewpoint of constraint relaxation using eye movement analysis. In Proceedings of the 4 th international conference of cognitive science, pages 671-676, 2003.

24. H. Terai and K. Miwa. Sudden and gradual processes of insight problem solving: Investigation by combination of experiments and simulations. In Proceedings of 28th annual meeting of the cognitive science society, pages 834-839, 2006.

25. G. Wallas. The art of thought. New York: Harcourt Brace Jovanovich, 1926.

26. R. W. Weisberg. Prolegomena to theories of insight in problem solving: A taxonomy of problems. In R. J. Sternberg and J. E. Davidson, editors, The nature of insight, chapter 5, pages 157-196. Cambridge, MA: MIT Press, 1995.

27. W. A. Wickelgren. How to solve problems. Sanfracisco: Freeman, 1974.

\section{Appendix: Solution of the insight tasks}

\section{Candle-box problem}

Solution: Empty the tackbox, and tack it to the wall. Set the candle on the platform formed by the box.

\section{Four chain problem}

Solution: Separate one of the four chains to three rings (cost: 6 cents). Connect the remaining three chains to each other by using three rings (cost: 9 cents).

\section{Remote Associate Test}

Solution: writer (typewriter, ghostwriter, storywriter)

\section{Nine dots problem}

Solution: See Fig. 5 .

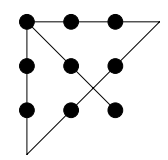

Fig. 5. Solution of nine dots problem

\section{Match stick problem}

Solution: See Fig. 6. Decompose IV to I and V, and move I from the left side of $\mathrm{V}$ to the right side.

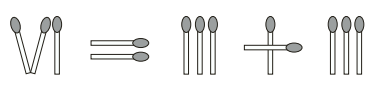

Fig. 6. Solution of match stick problem 